\title{
Discriminating Subtypes of Mild Cognitive Impairment according to Performance on Semantic Verbal Fluency Tasks with Different Administration Time
}

\author{
Soo Ryon Kim \\ Department of Speech and Hearing Therapy, College of Health Sciences, Catholic University of Pusan, Busan, Korea
}

의미 구어유창성 과제의 실시시간에 따른 경도인지장애 하위유형의 변별력

김 수 련

부산가톨릭대학교 보건과학대학 언어청각치료학과

\begin{abstract}
Purpose: Semantic (e.g., animal category) verbal fluency (SVF) tasks are widely utilized to measure subtle cognitive and language impairment caused by aging and neurological diseases. Mild cognitive impairment (MCI) regarded as transitional stage in aging with increased risk of incident dementia might show differential performances on SVF tasks according to $\mathrm{MCl}$ subtypes and different administration time. Methods: Three $\mathrm{MCl}$ subtypes of amnestic single-domain $\mathrm{MCl}$ (asMCI), amnestic multi-domain $\mathrm{MCl}$ (amMCI), non-amnestic MCI (non-aMCI), and age- and education-matched normal elderly (NE) participated in this study. Both the SVF tasks with 60- and 30-second administration time were conducted to four groups. Results: In 60-second SVF tasks, patients with amMCI and non-aMCI exhibited significantly lower performances than NE, but asMCI did not. In contrast, no significant differences were observed in 30-second SVF tasks between the groups. In receiver operating characteristics (ROC) curve analysis, 60-second SVF task showed fair discriminant power between amMCl and NE [area under ROC curve $(A \cup C)=0.779$ ] as well as between non-aMCl and NE $(A \cup C=0.733)$. Conclusion: The results indicated that subtle cognitive decline of $\mathrm{MCl}$ might not be revealed during short administration time because automatic access for lexico-semantic process during the initial phase of SVF task is relatively preserved. The present study suggests that 60 -second SVF tasks are useful to differentiate amMCl and non-aMCl groups from $\mathrm{NE}$, but not asMCI.
\end{abstract}

Key Words: Mild cognitive impairment, Semantic verbal fluency task, Administration time, Discriminant power.

Received: October 11, 2017 / Revised: October 19, 2017 / Accepted: October 20, 2017

Correspondence: Soo Ryon Kim, Department of Speech and Hearing Therapy, College of Health Sciences, Catholic University of Pusan, 57 Oryundaero, Geumjeong-gu, Busan 46252, Korea

Tel: +82-51-510-0846 / Fax: +82-51-510-0848 / E-mail: azzigom@naver.com

\section{INTRODUCTION}

구어유창성(verbal fluency, VF) 과제는 초기 알츠하이머치 매(dementia of alzheimer's type, DAT)나 경도인지장애(mild cognitive impairment, $\mathrm{MCI}$ )와 같은 인지·언어능력의 손상을 감별하는 데 매우 민감한 과제로 알려져 있다(Cooper et al., 2004; Forbes-McKay et al., 2005). VF 과제를 통해 집행기능, 의미기억, 작업기억 및 어휘-의미론적 처리과정 등의 문제를 확인할 수 있기 때문이다. $\mathrm{VF}$ 를 평가하는 가장 대표적인 방법
은 제한된 시간 내에 특정 범주나 글자로 시작하는 단어를 최 대한 많이 말하도록 하는 것이다. 특정 의미 범주(예: 동물)를 제시하는 의미 구어유창성(semantic verbal fluency, SVF) 과 제와 특정 글자나 음소(예: ‘ᄀ')를 제시하는 음소 구어유창성 (phonetic verbal fluency, PVF) 과제가 있다. 일반적으로 PVF 과제는 자소와 음소의 대응 지식, 음운 인식, 글자 체계에 대한 전략적 탐색 등과 같이 문해능력과 밀접한 관련이 있다. 따라서 PVF 과제는 대상자의 교육수준의 영향을 크게 받는다. 반면 $\mathrm{SVF}$ 과제는 연령 변인, 즉 노화에 따른 인지능력의 변화에 더 
민감하다(Tombaugh et al., 1999).

$\mathrm{MCI}$ 는 정상 노화와 치매의 중간 단계이다. 매년 일반노인의 1 2\%가 DAT로 진행되는 반면 MCI는 매년 10 15\%, 6년 이내 에 80\%가 DAT로 진행되는 치매 고위험군이다(Petersen et al., 2001). MCI는 기억력 손상의 유무와 손상된 인지영역의 개수 에 따라 다양한 하위유형으로 구분된다(Petersen, 2004). 먼저 기억력 손상이 두드러지는 기억상실형 경도인지장애(amnestic mild cognitive impairment, aMCI)는 DAT로의 진행 가능성이 높다. $\mathrm{aMCl}$ 는 기억력만 손상된 경우 단일영역 기억상실형 경도 인지장애(amnestic single-domain mild cognitive impairment, $\mathrm{asMCI}$, 기억력과 다른 인지기능이 복합적으로 손상된 경우 다 영역 기억상실형 경도인지장애(amnestic multiple-domain mild cognitive impairment, amMCI)로 분류된다. 기억력 외의 다른 인지기능이 손상된 경우 비기억상실형 경도인지장애(non-amnestic mild cognitive impairment, non-aMCI)로 분류되며, 이 유형은 레비소체치매(dementia with lewy bodies)나 전두 측두엽 변성(frontotemporal degeneration)과 같은 비알츠하이 머형 치매로의 진행 가능성이 높다(Fischer et al., 2007). 이렇듯 $\mathrm{MCI}$ 는 다양한 인지손상 양상으로 인해 이질적 성격을 보이는 집단이다(Devanand et al., 2010; Jefferson et al., 2006; Weakley et al., 2013).

Mirandez et al.(2017)의 조사에 따르면 2004년 이후로 MCI 환자를 대상으로 $\mathrm{VF}$ 과제를 실시한 연구는 19편이었다. 이 중 17 편이 SVF 과제를 활용하였고, 의미 범주 중 동물 범주를 포 함한 연구가 3편, 채소 범주 1편, 교통수단 1편, 도구 1편, 고유 명사 1 편, 가게에 있는 물건 1편이 있었다. SVF 과제에서 가장 많이 활용되는 의미 범주는 동물 범주이다. 그 이유는 동물 범 주는 다른 의미 범주(예: 채소, 도구)에 비하여 성별 및 교육수 준의 영향을 적게 받고(Capitani et al., 1999), 노화 및 신경학 적 질환으로 인한 인지·언어 능력의 변화를 잘 보여주기 때문 이다(Adlam et al., 2006; Lee et al., 2015; Troyer, 2000). 따라 서 한국 노인 집단과 같이 교육수준이 낮거나 개인 간 교육수 준의 차이가 큰 대상자에게(Korean Educational Development Institute, 2002) 동물 범주 SVF 과제는 특히 유용하다.

$\mathrm{MCI}$ 하위유형의 $\mathrm{VF}$ 과제의 수행 특성은 주로 $\mathrm{aMCI}$ 집단을 대상으로 보고되었다. 대부분의 선행연구는 $\mathrm{aMCI}$ 집단이 VF 과제에서 일반노인에 비해 저하된 수행력을 보인다고 하였다 (Choi, 2011; Jang \& Kim, 2014; Malek-Ahmadi et al., 2011; Murphy et al., 2006; Nutter-Upham et al., 2008; Price et al., 2012). 그러나 일반노인과 차이를 보이지 않는다는 상반된 연구 결과도 있다(Traykov et al., 2007). MCI 하위유형을 asMCI, $\mathrm{amMCI}$, non-aMCI로 세분화한 선행연구에서도 상이한 연구 결과가 혼재하고 있다. Weakley et al.(2013)은 asMCI와 non-
$\mathrm{aMCI}$ 의 SVF 과제 수행력은 정상군과 차이가 없었으나 $\mathrm{amMCI}$ 는 유의하게 저조하였다고 보고하였다. 반면 Brandt \& Man$\operatorname{ning}(2009)$ 은 asMCI만 차이가 없고 amMCI와 non-aMCI는 정상군과 차이가 있다고 보고하였다. 상이한 선행연구 결과와 함께 다양한 $\mathrm{MCI}$ 하위유형을 대상으로 SVF 과제의 수행 특성 을 보고한 연구는 여전히 부족한 실정이다.

일반적으로 SVF 과제는 제한시간 60초 동안 특정 범주에 속하는 단어를 최대한 많이 말하도록 한다. 그러나 최근 다양한 집단을 대상으로 60 초가 아닌 30초 SVF 과제의 유용성을 확인 하고 있다(Kim et al., 2011; Lee et al., 2015). 60초의 과제 실시 시간이 긴 것은 아니지만 신경학적 손상을 확인하는 평가과정 에 많은 과제들이 포함되므로 대상자들의 과제 수행에 대한 신 체적, 인지적 부담감은 증가하게 된다. 과제 실시시간의 단축은 피검자의 과제에 대한 주의력을 향상시켜 줄 뿐만 아니라, 검사 결과의 양적, 질적 분석에 대한 검사자의 부담감을 감소시켜 준 다. 노화에 따른 인지·언어 능력의 변화를 확인하기 위해 실시 한 30초 SVF 과제는 5년 연령단위의 일반노인 집단 간 차이를 유의하게 보여주었다(Lee et al., 2015). 또한 뇌졸중 실어증 환자, 뇌졸중 비실어증 환자, 정상노인 간에도 유의한 차이를 보여 30 초의 짧은 과제 실시시간이 유용함을 제시하였다(Kim et al., 2011). MCI 환자의 경우 30초 SVF 과제를 직접 실시하지는 않 았으나, 60초 SVF 과제의 수행 결과 중 초기 20 30초 시간 구 간을 분석한 연구들이 있다. $\mathrm{MCI}$ 집단을 일반노인 집단과 비교 하였을 때 MCI 집단의 과제 수행력이 유의하게 낮았다(Demetriou \& Holtzer, 2017). 반면 MCI를 asMCI, amMCI, non$\mathrm{aMCI}$ 의 세 하위유형으로 구분하여 비교하였을 때는 일반노인 집단과 유의한 차이를 보이지 않기도 하였다(Weakley et al., 2013). 이러한 상반된 연구 결과는 $\mathrm{MCI}$ 의 하위집단을 분류하여 30초 SVF 과제의 수행 특성을 살펴보아야 할 필요성을 제시한다. 이에 본 연구는 $\mathrm{MCI}$ 하위유형인 asMCI, amMCI, non-aMCI 집단과 일반노인 집단을 대상으로 60초 및 30초 SVF 과제 를 실시하여 집단 간 차이를 살펴보고, SVF 과제의 실시시간에 따른 $\mathrm{MCI}$ 와 일반노인 집단 간 변별력을 확인하고자 하였다.

\section{MATERIALS AND METHODS}

\section{연구 대상}

본 연구는 경기도 소재의 분당서울대학교병원 신경과에서 $\mathrm{MCI}$ 로 진단받은 환자 70명과 만 65 85세 일반노인 23명을 대 상으로 하였다. $\mathrm{MCI}$ 의 진단기준은 다음과 같았다. 신경과 전문 의로부터 Petersen(2004)의 진단 기준에 따라 환자 본인 혹은 보호자가 주관적 기억력 손상을 보고하고, 연령을 고려한 객관 적 기억장애가 확인되지만, 전반적인 인지기능은 정상범위에 
속하여 일상생활에 큰 어려움이 없으며, 치매가 아닌 경우이다. 임상치매척도(clinical dementia rating, CDR) (Hughes et al., 1982)에서 0.5점에 해당하였으며, 한국판 노인우울척도 단축형 (geriatric depression scale short form-Korean version, GDSSFK) $(\mathrm{Kee}, 1996)$ 실시 결과 우울증이 없었다.

$\mathrm{MCI}$ 집단은 서울신경심리검사(Seoul neuropsychological screening battery, SNSB) (Kang \& Na, 2003) 결과의 프로파 일에 근거하여 세 하위유형으로 분류되었다. 첫째, $\mathrm{asMCI}$ 는 언 어적 또는 비언어적 기억력 평가 항목의 지연회상 능력이 $16 \%$ ile 미만의 수행을 보였다. 둘째, amMCI는 기억력뿐만 아니라 다른 인지능력 저하를 동반하였다. 셋째, non-aMCI는 기억력 외의 다른 인지기능의 손상을 보였다(Petersen, 2004).

일반노인의 기준은 다음과 같았다. 건강선별설문지(Christensen et al., 1991)를 실시하여 신체기능이 정상으로 판단되었 으며, 한국판 간이정신상태검사(Korean version of mini-mental state examination, K-MMSE) (Kang et al., 1997)를 실시 하여 연령과 교육수준을 기준으로 $16 \%$ tile 이상의 수행을 보인 경우 대상자에 포함하였다(Kang, 2006). 그리고 GDSSF-K (Kee, 1996)를 실시하여 우울증이 없음을 확인하였다.

네 집단 간 대상자 성비에 차이가 있는지를 알아보기 위해 카이제곱검정(chi-square test)을, 연령, 교육 연수에 차이가 있 는지를 확인하기 위해 일원분산분석(one-way ANOVA)을 실시 하였다. 그 결과 성비 $\left(\chi^{2}=2.848, p>0.05\right)$, 연령 $\left[\mathrm{F}_{(3,89)}=1.868\right.$, $p>0.05]$, 교육 연수 $\left[\mathrm{F}_{(3,89)}=1.405, p>0.05\right]$ 에서 유의한 차이가 없었다. MCI 하위유형과 일반노인 집단의 대상자 정보는 Table 1 과 같다. 본 연구는 생명윤리심의위원회의 승인을 받아 실시하 였다(IRB\#: B-1402-240-106).

\section{연구 절차}

$\mathrm{MCI}$ 환자는 외래 내원 시 60초 SVF 과제를 실시하였고, 1 2 개월 후 재방문하여 30 초 SVF 과제를 실시하였다. 일반노인 은 복지관에서 면담을 진행하며 인구학적 정보 및 건강상태에 관한 설문지를 작성하도록 하였다. 전반적인 인지능력을 확인하 기 위해 K-MMSE를 실시한 후 60초 SVF 과제를 실시하였다. 약 1 개월 후 동일 노인을 대상으로 30 초 SVF 과제를 실시하였
다. 모든 검사는 조용한 방에서 검사자와 일대일로 진행되었으 며, 검사자는 대상자가 과제 수행 시 표현한 모든 반응을 검사 지에 실시간으로 기록하고 녹음하였다. 녹음된 자료를 바탕으 로 대상자의 반응을 최종 확인하여 정리하였다.

\section{통계 분석}

집단 간 SVF 과제의 점수 차이를 확인하기 위해 일원분산분 석(one-way ANOVA)을 실시하였고, 사후분석은 Tukey를 이 용하였다. SVF 과제가 집단 간 차이를 변별시켜 주는지를 확인 하기 위해 이원로지스틱회귀(binary logistic regression) 분석을 실시한 후 수신자 조작 특성(receiver operating characteristics, ROC) 곡선을 통해 절단점(cut-off score), 민감도(sensitivity), 특이도(specificity) 및 곡선하 면적(area under ROC curve, $\mathrm{AUC})$ 을 살펴보았다. 모든 통계처리는 통계분석 패키지인 윈도 우용 SPSS 24.0 (IBM Corp., Armonk, NY, USA)을 사용하였다.

\section{RESULTS}

\section{집단 간 의미 구어유창성 과제의 점수 비교}

asMCI, amMCI, non-aMCI와 일반노인의 네 집단 간 60초 $\mathrm{SVF}$ 과제의 점수 차이에 대한 일원분산분석 결과, 집단 간 차 이는 유의하였다 $\left[\mathrm{F}_{(3,89)}=3.857, p=0.012\right]$. 사후검정 결과 $\mathrm{amMCI}$ 와 일반노인 집단 간, non-aMCI와 일반노인 집단 간 차 이는 유의하였으나, 그 외 집단 간 차이는 유의하지 않았다. 60 초 SVF 과제 점수는 amMCI와 non-aMCI 집단이 일반노인 집 단보다 유의하게 낮았다.

다음으로 네 집단 간 30초 SVF 과제의 점수 차이에 대한 일 원분산분석을 실시한 결과, 집단 간 차이는 유의하지 않았다 $\left[\mathrm{F}_{(3,89)}=1.687, p=0.176\right]$. 60초와 30초 SVF 과제의 점수 평균, 표준편차 및 일원분산분석 결과는 Table 2 와 같다.

\section{의미 구어유창성 과제의 집단 변별력}

60초 SVF 과제가 amMCI와 일반노인 집단 간, non-aMCI 와 일반노인 집단 간 집단 변별력을 갖고 있는지 확인하기 위해 이원로지스틱회귀분석을 실시하였다. 그 결과 60 초 SVF 과제

Table 1. Participants' characteristics

\begin{tabular}{llccc}
\hline \multicolumn{1}{c}{ Groups } & $\mathrm{n}(\mathrm{M}: \mathrm{F})$ & Age $(\mathrm{yr})$ & Education $(\mathrm{yr})$ & K-MMSE \\
\hline asMCI & $23(10: 13)$ & $73.39(8.40)$ & $12.61(4.09)$ & $26.57(1.75)$ \\
amMCI & $24(8: 16)$ & $73.75(5.40)$ & $13.04(4.76)$ & $25.92(2.30)$ \\
non-aMCI & $23(11: 12)$ & $69.35(9.24)$ & $10.82(4.16)$ & $27.17(1.85)$ \\
NE & $23(6: 17)$ & $73.00(4.70)$ & $11.39(3.63)$ & $27.96(2.26)$ \\
\hline
\end{tabular}

Values are presented as mean (standard deviation). M: male, F: female, asMCI: amnestic single-domain mild cognitive impairment, amMCI: amnestic multiple-domain mild cognitive impairment, non-aMCI: non-amnestic mild cognitive impairment, NE: normal elderly, K-MMSE: Korean version of mini-mental state examination (Kang et al., 1997). 
는 유의한 예측력(predictive power)을 보였다(Table 3). 다음으 로 $\mathrm{ROC}$ 곡선 분석을 실시한 결과, 60 초 $\mathrm{SVF}$ 과제는 $\mathrm{amMCI}$ 와 일반노인의 경우 절단점 13점을 기준으로 민감도 $66.67 \%$, 특이도 $86.96 \%$, AUC 0.779를 보였다. non-aMCI와 일반노인 의 경우에는 절단점 12 점을 기준으로 민감도 $60.87 \%$, 특이도 91.30\%, AUC 0.733을 보였다(Figure 1).

\section{DISCUSSIONS}

본 연구는 MCI 하위집단을 대상으로 60초와 30초 SVF 과제 를 실시하여 집단 간 수행력을 비교하였다. 집단 간 유의한 차이 를 보인 과제의 경우 이원로지스틱회귀분석과 $\mathrm{ROC}$ 곡선 분석 을 통해 집단 변별력을 살펴보았다.

연구 결과 60 초 $\mathrm{SVF}$ 과제에서 $\mathrm{amMCI}$ 와 non-aMCI 집단은 일반노인보다 유의하게 낮은 점수를 획득하였으나 $\mathrm{asMCI}$ 는 일
반노인과 비슷한 수행을 보였다. 이러한 MCI 하위유형 간 차이 는 $\mathrm{MCI}$ 의 다양한 인지손상 양상과 SVF 과제의 특성으로 설 명될 수 있다. asMCI는 인지영역 중 기억력 손상만 두드러지는 반면, amMCI와 non-aMCI는 기억력 외의 다른 인지기능 손 상을 동반하고 있다. SVF 과제는 특정 의미 범주에 포함된 어 휘에 대한 의미기억뿐만 아니라 집행기능, 주의력, 전반적 모니 터링능력, 작업기억 등 다양한 인지능력을 요구한다(Chertkow $\& \mathrm{Bub}, 1990)$. 즉, SVF 과제를 잘 수행하기 위해서는 회상을 조직화하는 전략(예: 가축, 바다 동물, 조류, 포유동물 등)을 세 우고, 언어적 측면에서 의미지식, 어휘인출, 음운 부호화, 조음과 같은 과정을 처리해야 한다(Radanovic et al., 2009). SVF 과제 가 의미기억뿐만 아니라 집행기능과 같은 인지기능을 모두 요구 하는 과제이긴 하지만, 기억력 손상만 있는 $\mathrm{asMCI}$ 집단이 정상 군과 비슷한 수행력을 보였다는 것은 SVF 과제 수행에 의미기 억보다는 의미기억의 효율적 처리에 요구되는 다른 인지능력들

Table 2. Comparison of semantic verbal fluency task score between $\mathrm{MCl}$ subtypes and normal elderly

\begin{tabular}{|c|c|c|c|c|c|c|}
\hline & \multicolumn{4}{|c|}{ Mean (SD) } & \multirow{2}{*}{$\mathrm{F}$} & \multirow{2}{*}{ Tukey post hoc } \\
\hline & asMCI & amMCI & non-aMCI & $\mathrm{NE}$ & & \\
\hline \multicolumn{7}{|l|}{ SVF tasks } \\
\hline 60-second & $14.30(4.22)$ & $12.58(3.90)$ & $13.04(4.15)$ & $15.96(2.16)$ & $3.857^{*}$ & $\begin{array}{l}\text { amMCI }<\mathrm{NE}^{*} \\
\text { non-aMCI }<\mathrm{NE}^{*}\end{array}$ \\
\hline 30-second & $9.26(2.88)$ & $8.79(2.87)$ & $9.35(3.18)$ & $10.52(1.78)$ & 1.687 & \\
\hline
\end{tabular}

${ }^{*} p<0.05$. MCI: mild cognitive impairment, SVF: semantic verbal fluency, SD: standard deviation, asMCI: amnestic single-domain mild cognitive impairment, amMCI: amnestic multiple-domain mild cognitive impairment, non-aMCI: non-amnestic mild cognitive impairment, NE: normal elderly

Table 3. Results of the binary logistic regression analysis

\begin{tabular}{|c|c|c|c|c|c|c|c|c|}
\hline & \multirow{2}{*}{ B } & \multirow{2}{*}{ SE } & \multirow{2}{*}{ Wald } & \multirow{2}{*}{ df } & \multirow{2}{*}{ Sig } & \multirow{2}{*}{$\operatorname{Exp}(B)$} & \multicolumn{2}{|c|}{ 95\% CI for $\operatorname{Exp}(B)$} \\
\hline & & & & & & & Lower & Upper \\
\hline amMCI vs. NE & -0.341 & 0.116 & 8.597 & 1 & 0.003 & 0.711 & 0.566 & 0.893 \\
\hline non-aMCI vs. NE & -0.265 & 0.102 & 6.800 & 1 & 0.009 & 0.767 & 0.628 & 0.936 \\
\hline
\end{tabular}

amMCI: amnestic mild cognitive impairment, non-aMCI: non-amnestic mild cognitive impairment, NE: normal elderly, B: logistic coefficient, SE: standard error of estimate, Wald: Wald chi-square values, df: degree of freedom, Sig : significance, Exp (B): exponentiated coefficient, $95 \%$ CI for EXP (B): 95\% confidence interval for $\operatorname{Exp}(B)$

Figure 1. ROC curve evaluation of 60 second verbal fluency task for $\mathrm{MCl}$ diagnosis. ROC: receiver operating characteristics, MCl: mild cognitive impairment, amMCl: amnestic multiple-domain mild cognitive impairment, non-aMCl: non-amnestic mild cognitive impairment, AUC: area under ROC curve.
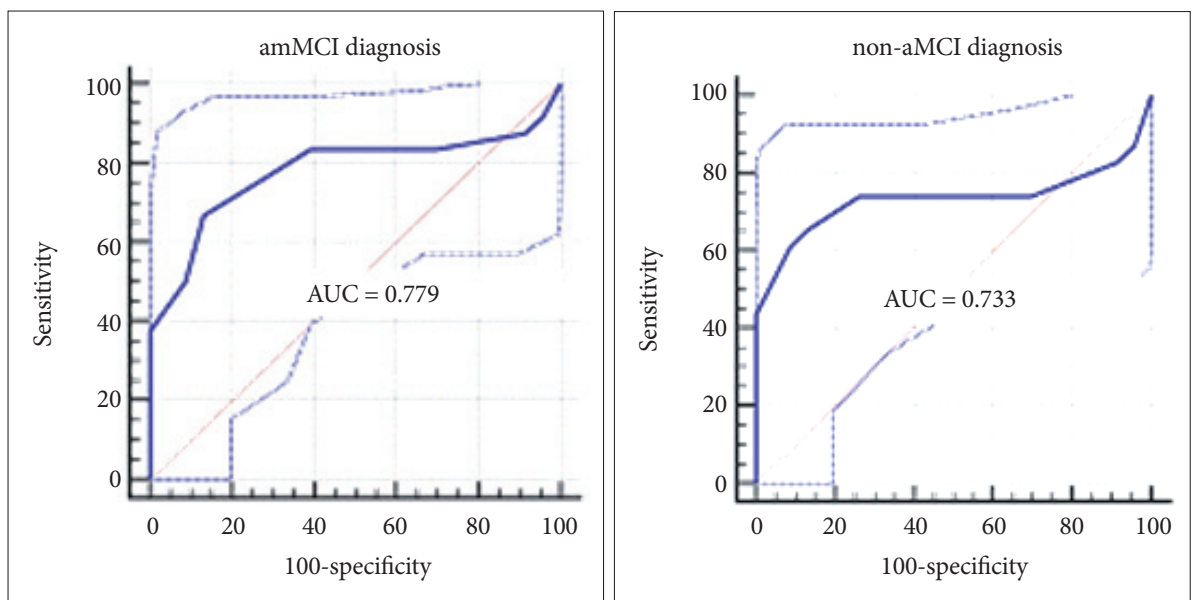
이 더 크게 작용한다는 것을 의미한다(Weakley et al., 2013).

$\mathrm{aMCI}$ 집단을 대상으로 한 다수의 선행연구들(Choi, 2011; Jang \& Kim, 2014; Malek-Ahmadi et al., 2011; Murphy et al., 2006; Nutter-Upham et al., 2008; Price et al., 2012)은 $\mathrm{SVF}$ 과제에서 $\mathrm{aMCI}$ 집단의 수행력이 손상되었음을 보고하였 다. 그러나 본 연구에서 $\mathrm{aMCI}$ 집단을 기억력만 손상된 $\mathrm{asMCI}$ 와 기억력과 다른 인지능력이 함께 손상된 $\mathrm{amMCI}$ 로 세분화하 여 비교하였을 때 정상군과의 차이를 보이는 것은 $\mathrm{amMCI}$ 집 단뿐이었다. $\mathrm{asMCI}$ 와 $\mathrm{amMCI}$ 의 구분 없이 전체 $\mathrm{aMCI}$ 집단을 대상으로 보고한 SVF 과제의 낮은 수행력은 $\mathrm{amMCI}$ 의 영향으 로 발생했을 가능성이 크다. 따라서 $\mathrm{MCI}$ 집단을 인지손상의 양상에 따라 세분화하여 그 특성을 살펴보는 것이 중요하다.

본 연구의 결과는 asMCI가 정상군과 차이가 없고 $\mathrm{amMCI}$ 가 유의한 차이를 보인다는 측면에서는 선행연구 결과와 일치하지 만, non-aMCI가 정상군과 유의한 차이를 보인다는 측면에서는 선행연구에 따라 다르다(Brandt \& Manning, 2009; Weakley et al., 2013). non-aMCI의 경우 Brandt \& Manning(2009)은 본 연구 결과와 마찬가지로 일반노인에 비해 낮은 수행력을 보 인다고 보고하였으나, Weakley et al.(2013)은 정상군과 유의한 차이를 보이지 않는다고 보고하였다. 이러한 상반된 결과에 대 해 연구자들은 non-aMCI 집단의 대상자 수(16명)가 다른 집 단(asMCI $=25$ 명, $\mathrm{amMCI}=49$ 명 $)$ 에 비해 적었기 때문에 결과 해석에 제한점이 있다고 논의하였다(Weakley et al., 2013). 뿐 만 아니라 $\mathrm{aMCI}$ 집단은 단일영역 손상과 다영역 손상으로 세 분화되었으나, non-aMCI 집단은 손상된 영역의 개수로 세분 화되지 않아 연구마다 이질적 특징을 보일 가능성이 크므로 연 구 결과가 상이할 수 있다.

둘째, 30초 SVF 과제에서 $\mathrm{MCI}$ 하위유형과 일반노인 집단 간에 유의한 차이는 나타나지 않았다. 선행연구에서 다양한 연 령대의 일반노인 집단 간(Lee et al., 2015) 및 뇌졸중 실어증 환 자와 통제군 간(Kim et al., 2011)에 관찰되었던 30초 SVF 과 제의 수행 차이는 $\mathrm{MCI}$ 하위유형을 대상으로 한 본 연구에서 는 나타나지 않았다. Demetriou \& Holtzer(2017)는 MCI 집단 이 산출한 60 초 SVF 과제의 반응을 20초 단위로 구분하여 각 시간단위의 수행을 분석하였다. 그 결과 $\mathrm{MCI}$ 환자는 첫 번째 20초 구간에서 정상집단보다 유의하게 적은 단어를 산출하였 다. Weakley et al.(2013)은 asMCI, amMCI, non-aMCI, 일반 노인을 대상으로 60 초 SVF 과제를 실시하고 30초 단위로 구분 하여 분석하였다. 그 결과 $\mathrm{MCI}$ 세 하위유형 모두 초기 30초 구 간에서는 일반노인과 유의한 차이가 없었다. 이러한 선행연구 의 결과는 $\mathrm{MCI}$ 하위유형 구분 여부에 따라 연구 결과에 차이 가 있음을 시사한다. 또한 30초 SVF 과제가 $\mathrm{MCI}$ 하위유형과 일반노인 집단 간 차이를 보여주지 못한다는 본 연구 결과와
일치한다.

선행연구의 60 초 SVF 과제의 초기 30초 구간과 본 연구의 30초 SVF 과제의 MCI 집단의 수행력에 대한 결과 차이는 다 음의 측면에서 살펴볼 수 있다. SVF 과제 실시의 초기 단계는 반자동화된 인출(semi-automatic retrieval) 과정이므로 익숙 하고 쉬운 단어들이 빠르게 인출된다. 이후 시간구간에서는 초 기에 쉽게 인출되는 단어들이 다 소진되기 때문에 의도적 인출 (effortful retrieval)이 요구된다(Crowe, 1998; Raboutet et al., 2010). SVF 과제의 어휘-의미 처리과정인 자동적 접근(automatic access)과 의도적 접근(intentional access) 중 $\mathrm{MCI}$ 환자들 은 의도적 접근이 먼저 손상되기 시작한다(Duong et al., 2006). 인지 손상이 크지 않은 $\mathrm{MCI}$ 환자의 경우 과제 수행의 초기 30 초 동안 일어나는 자동적 접근에 어려움이 없다. 반면 과제의 실시시간이 길어질수록 어휘인출 과정에서 의도적 접근이 더욱 요구되므로 과제의 후반부에서 수행력이 낮아지게 된다. 따라서 $\mathrm{MCI}$ 의 미세한 인지능력 손상을 보여주기 위해서는 60 초라는 충분한 시간이 제시될 필요가 있다. 또한 과제의 실시시간은 대 상자의 특성을 고려하여 적용되어야 함을 시사한다(Kim \& Kim, 2017).

마지막으로 amMCI와 일반노인 간, non-aMCI와 일반노인 간 유의한 수행 차이를 보인 60초 SVF 과제는 ROC 곡선 분석 결 과 두 경우 모두 AUC 0.70을 넘는 양호한 변별력(fair discriminant power)을 보였다. $\mathrm{MCI}$ 환자를 대상으로 SVF 과제의 절단 점을 보고한 국내연구를 살펴보면 다음과 같다. Jang \& Kim (2014)은 aMCI와 일반노인의 절단점을 11점으로 보고하였고, Choi(2011)는 aMCI와 정상고령자의 절단점을 12.5점으로 보고 하였다. 본 연구에서는 $\mathrm{amMCl}$ 와 일반노인의 절단점은 13 점, non-aMCI와 일반노인의 절단점은 12점이었다. 이러한 차이는 연구 대상자의 특성에서 기인한다. Jang \& Kim(2014)의 연구 에서 $\mathrm{MCI}$ 집단의 교육 연수는 1.78 년으로 매우 적었고, 일반노 인의 교육 연수 5.52년과 비교하였을 때 집단 간 차이가 컸다. 또한 대상자의 성별은 모두 여성이었다. Choi(2011)의 연구에서 는 정상고령자와 $\mathrm{MCI}$ 집단의 교육 연수가 각각 10.4년, 9.7년이 었다. 본 연구에서는 일반노인 11.39년, amMCI 13.04년, non$\mathrm{aMCI} 10.82$ 년으로 선행연구에 비해 교육 연수가 높았다. 세 연 구를 통해 교육 연수가 높은 대상자일수록 절단점이 높아지는 양상을 보였다. 교육수준은 대상자의 연령, 성별, 인지수준을 통 제하였을 때 SVF 과제 점수에 크게 영향을 미치는 변인이다 (Kawano et al., 2010). 또한 교육수준이 낮은(4 8년) MCI 집단에 서는 SVF 과제의 집단 변별력이 낮았지만, 교육수준이 높은(9 년 이상) 집단에서는 높은 변별력을 보였다(Radanovic et al., 2009). 따라서 SVF 과제의 결과 해석 시 대상자 집단의 교육수 준을 반드시 고려해야 한다. 
$\mathrm{MCI}$ 는 정상 노화와 치매의 중간 단계로 정의되고 있지만 인 지·언어 능력의 손상 양상이 매우 다양한 집단이므로 하위유형 을 구분하여 연구해야 한다. 임상적 효율성을 증진시키기 위해 단축형 과제들이 개발되고 있으나 평가 대상자의 특성을 반영 하여 과제의 유용성을 검증하여야 한다. 본 연구에서는 $\mathrm{MCI}$ 를 세 하위유형으로 분류하였고, 기존에 실시되고 있는 60 초 SVF 과제가 amMCI와 non-aMCI의 인지손상을 민감하게 평가할 수 있음을 제시하였다. 반면 단축형인 30초 SVF 과제는 MCI 하위유형 간 차이를 변별하는 데 유용하지 못하였다. 추후 연구 에서 동물 범주 외의 다양한 의미 범주 SVF 과제를 사용하여 의미 범주의 차이에 따른 $\mathrm{MCI}$ 수행력을 살펴볼 필요가 있다. 또 한 본 연구에서 한 집단으로 분류되었던 non-aMCI를 단일영역 과 다영역으로 재분류하거나 $\mathrm{aMCI}$ 의 기억력 손상을 등록(encoding) 손상과 인출(retrieval) 손상으로 재분류하여 더욱 세 분화된 $\mathrm{MCI}$ 하위유형의 인지·언어적 특성에 대한 정보를 제공 할 수 있을 것이다.

중심 단어 : 경도인지장애·의미 구어유창성·과제 실시시간· 집단 변별력.

\section{Acknowledgments}

본 논문은 2016년 부산가톨릭대학교 교내학술연구비 지원에 의하 여 수행된 것임(2016-1-057).

\section{REFERENCES}

Adlam, A.L., Bozeat, S., Arnold, R., Watson, P., \& Hodges, J. R. (2006). Semantic knowledge in mild cognitive impairment and mild Alzheimer's disease. Cortex, 42(5), 675-684.

Brandt, J. \& Manning, K. J. (2009). Patterns of word-list generation in mild cognitive impairment and Alzheimer's disease. The Clinical Neuropsychologist, 23(5), 870-879.

Capitani, E., Laiacona, M., \& Barbarotto, R. (1999). Gender affects word retrieval of certain categories in semantic fluency tasks. Cortex, 35(2), 273-278.

Chertkow, H. \& Bub, D. (1990). Semantic memory loss in dementia of Alzheimer's type. What do various measures measure? Brain, 113(Pt 2), 397-417.

Choi, H. (2011). Detecting language deficits in patients with mild cognitive impairment through verbal fluency and picture description tasks. Korean Journal of Communication Disorders, 16(2), 171-184.

Christensen, K. J., Multhaup, K. S., Nordstrom, S., \& Voss, K. (1991). A cognitive battery for dementia: Development and measurement characteristics. Psychological Assessment: A Journal of Consulting and Clinical Psychology, 3(2), 168-174.

Cooper, D. B., Lacritz, L. H., Weiner, M. F., Rosenberg, R. N., \& Cullum, C. M. (2004). Category fluency in mild cognitive impairment: Reduced effect of practice in test-retest conditions. Alzheimer Disease and Associated Disorders, 18(3), 120-122.

Crowe, S. F. (1998). Decrease in performance on the verbal fluency test as a function of time: Evaluation in a young healthy sample. Journal of Clinical Experimental Neuropsychology, 20(3), 391-401.

Demetriou, E. \& Holtzer, R. (2017). Mild cognitive impairments moderate the effect of time on verbal fluency performance. Journal of the International Neuropsychological Society, 23(1), 44-55.
Devanand, D. P., Tabert, M. H., Cuasay, K., Manly, J. J., Schupf, N., Brickman, A. M., et al. (2010). Olfactory identification deficits and MCI in a multi-ethnic elderly community sample. Neurobiology of Aging, 31(9), 1593-1600.

Duong, A., Whitehead, V., Hanratty, K., \& Chertkow, H. (2006). The nature of lexico-semantic processing deficits in mild cognitive impairment. Neuropsychologia, 44(10), 1928-1935.

Fischer, P., Jungwirth, S., Zehetmayer, S., Weissgram, S., Hoenigschnabl, S., Gelpi, E., et al. (2007). Conversion from subtypes of mild cognitive impairment to Alzheimer dementia. Neurology, 68(4), 288-291.

Forbes-McKay, K. E., Ellis, A. W., Shanks, M. F., \& Venneri, A. (2005). The age of acquisition of words produced in a semantic fluency task can reliably differentiate normal from pathological age related cognitive decline. Neuropsychologia, 43(11), 1625-1632.

Hughes, C. P., Berg, L., Danziger, W. L., Coben, L. A., \& Martin, R. L. (1982), A new clinical scale for the staging of dementia. The British Journal of Psychiatry, 140, 566-572.

Jang, S. W. \& Kim, J. W. (2014). Detecting language performance in patients with amnestic mild cognitive impairment. Journal of SpeechLanguage \& Hearing Disorders, 23(4), 23-32.

Jefferson, A. L., Wong, S., Bolen, E., Ozonoff, A., Green, R. C., \& Stern, R. A. (2006). Cognitive correlates of HVOT performance differ between individuals with mild cognitive impairment and normal controls. Archives of Clinical Neuropsychology, 21(5), 405-412.

Kang, Y. (2006). A normative study of the Korean-mini mental state examination (K-MMSE) in the elderly. Korean Journal of Psychology: General, 25(2), 1-12.

Kang, Y. \& Na, D. L. (2003). Seoul Neuropsychological Screening Battery (SNSB). Seoul: Human Brain Research \& Consulting Co.

Kang, Y., Na, D. L., \& Hahn, S. (1997). Korean Version of Mini-Mental State Examination (K-MMSE). Seoul: Human Brain Research \& Consulting Co.

Kawano, N., Umegaki, H., Suzuki, Y., Yamamoto, S., Mogi, N., \& Iguchi, A. (2010). Effects of educational background on verbal fluency task performance in older adults with Alzheimer's disease and mild cognitive impairment. International Psychogeriatrics, 22(6), 995-1002.

Kee, B. S. (1996). A preliminary study for the standardization of geriatric depression scale short form-Korea version. Journal of the Korean Neuropsychiatric Association, 35(2), 298-307.

Kim, H., Kim, J., Kim, D. Y., \& Heo, J. (2011). Differentiating between aphasic and nonaphasic stroke patients using semantic verbal fluency measures with administration time of 30 seconds. European Neurolo$g y, 65(2), 113-117$.

Kim, S. R. \& Kim, H. (2017). Administration methods of word definition task for normal elderly. Journal of the Korean Data Analysis Society, 19(2), 971-980.

Korean Educational Development Institute (2002). Study on Illiteracy of Korean Elderly. Seoul: Korean Educational Development Institute.

Lee, S. H., Kim, H., Kim, J., Yoon, J. H., \& Kim, S. R. (2015). Initial phase performance in a 30-s verbal fluency task as being reflective of aging effect. Geriatrics and Gerontology International, 15(4), 496-500.

Malek-Ahmadi, M., Small, B. J., \& Raj, F. (2011). The diagnostic value of controlled oral word association test-FAS and category fluency in single-domain amnestic mild cognitive impairment. Dementia and Geriatric Cognitive Disorders, 32(4), 235-240.

Mirandez, R. M., Aprahamian, I., Talib, L. L., Forlenza, O. V., \& Radanovic, M. (2017). Multiple category verbal fluency in mild cognitive impairment and correlation with CSF biomarkers for Alzheimer's disease. International Psychogeriatrics, 29(6), 949-958.

Murphy, K. J., Rich, J. B., \& Troyer, A. K. (2006). Verbal fluency patterns in amnestic mild cognitive impairment are characteristic of Alzheimer's type dementia. Journal of the International Neuropsychological Society, 12(4), 570-574.

Nutter-Upham, K. E., Saykin, A. J., Rabin, L. A., Roth, M. R., Wishart, H. A., Pare, N., et al. (2008). Verbal fluency performance in amnestic MCI 
and older adults with cognitive complaints. Archives of Clinical Neuropsychology, 23(3), 229-241

Petersen, R. C. (2004). Mild cognitive impairment as a diagnostic entity. Journal of International Medicine, 256(3), 183-194.

Petersen, R. C., Doody, R., Kurz, A., Mohs, R. C., Morris, J. C., Rabins, P. V., et al. (2001). Current concepts in mild cognitive impairment. Archives of Neurology, 58(12), 1985-1992.

Price, S. E., Kinsella, G. J., Ong, B., Storey, E., Mullaly, E., Phillips, M., et al. (2012). Semantic verbal fluency strategies in amnestic mild cognitive impairment. Neuropsychology, 26(4), 490-497.

Raboutet, C., Sauzéon, H. Corsini, M. M., Rodrigues, J., Langevin, S., \& N'Kaoua, B. (2010). Performance on a semantic verbal fluency task across time: Dissociation between clustering, switching, and categori$\mathrm{cal}$ exploitation processes. Journal of Clinical and Experimental Neuropsychology, 32(3), 268-280.

Radanovic, M., Diniz, B. S., Mirandez, R. M., Novaretti, T. M., Flacks, M.
K., Yassuda, M. S., et al. (2009). Verbal fluency in the detection of mild cognitive impairment and Alzheimer's disease among Brazilian Portuguese speakers: The influence of education. International Psychogeriatrics, 21(6), 1081-1087.

Tombaugh, T. N., Kozak, J., \& Rees, L. (1999). Normative data stratified by age and education for two measures of verbal fluency: FAS and animal naming. Archives of Clinical Neuropsychology, 14(2), 167-177.

Traykov, L., Raoux, N., Latour, F., Gallo, L., Hanon, O., Baudic, S., et al. (2007). Executive functions deficit in mild cognitive impairment. Cognitive and Behavioral Neurology, 20(4), 219-224.

Troyer, A. K. (2000). Normative data for clustering and switching on verbal fluency tasks. Journal of Clinical and Experimental Neuropsychology, 22(3), 370-378.

Weakley, A., Schmitter-Edgecombe, M., \& Anderson, J. (2013). Analysis of verbal fluency ability in amnestic and non-amnestic mild cognitive impairment. Archives of Clinical Neuropsychology, 28(7), 721-731. 